\title{
Autophagy enhancement contributes to the synergistic effect of vitamin $D$ in temozolomide-based glioblastoma chemotherapy
}

\author{
DONG-HO BAK ${ }^{1}$, SEONG HEE KANG ${ }^{2}$, DU RI CHOI ${ }^{1}$, MI NA GIL ${ }^{1}$, KWANG SIK YU ${ }^{1}$, JI HEUN JEONG ${ }^{1}$, \\ NAM-SEOB LEE ${ }^{1}$, JE-HUN LEE ${ }^{1}$, YOUNG-GIL JEONG ${ }^{1}$, DONG KWAN KIM ${ }^{3}$, \\ DO-KYUNG KIM ${ }^{4}$, JWA-JIN KIM ${ }^{1,5}$ and SEUNG-YUN HAN ${ }^{1,5}$
}

\begin{abstract}
Departments of ${ }^{1}$ Anatomy, ${ }^{2}$ Radiological Science and ${ }^{3}$ Physiology, College of Medicine, Konyang University; ${ }^{4}$ Industry Cooperation Foundation, Konyang University; ${ }^{5}$ Myunggok Research Institute, College of Medicine, Konyang University, Daejeon 302-718, Republic of Korea
\end{abstract}

Received March 16, 2015; Accepted January 15, 2016

DOI: $10.3892 / e t m .2016 .3196$

\begin{abstract}
Temozolomide (TMZ), an alkylating agent, is recommended as the initial treatment for high-grade glioblastoma. TMZ is widely used, but its short half-life and the frequency of tumor resistance limit its therapeutic efficacy. In the present study, the anticancer effect of vitamin D (VD) combined with TMZ upon glioblastoma was determined, and the underlying mechanism of this effect was identified. Through cell viability, clonogenic and wound healing assays, the current study demonstrated that treatment of a C6 glioblastoma cell line with TMZ and VD resulted in significantly increased in vitro antitumor effects compared with either VD or TMZ alone. Autophagy, hypothesized to be the dominant mechanism underlying TMZ-based tumor cell death, was maximally activated in TMZ and VD co-treated C6 cells. This was demonstrated by ultrastructural observations of autophagosomes, increased size and number of microtubule-associated protein 1 light chain 3 (LC3) puncta and increased conversion of LC3-I to LC3-II. However, the extent of apoptosis was not significantly different between cells treated with TMZ and VD and those treated with TMZ alone. Addition of the autophagy inhibitor 3-methyladenine markedly inhibited the anticancer effect of TMZ and VD treatment, indicating that the chemosensitizing effect of VD in TMZ-based glioblastoma therapy is generated through enhancement of cytotoxic autophagy. TMZ and VD co-treatment also significantly inhibited tumor progression and prolonged survival duration in rat glioblastoma orthotopic xenograft models when compared with TMZ treatment alone. These in vivo results are concordant with the aforementioned in vitro results, together revealing that the
\end{abstract}

Correspondence to: Professor Seung-Yun Han or Dr Jwa-Jin Kim, Department of Anatomy, College of Medicine, Konyang University, 685 Gasuwon-dong, Daejeon 302-718, Republic of Korea

E-mail: jjzzy@konyang.ac.kr

E-mail: kjj1021@konyang.ac.kr

Key words: temozolomide, vitamin D, glioblastoma, autophagy, orthotopic xenograft combined use of TMZ and VD exerts synergistic antitumor effects on rat models of glioblastoma and may represent an effective therapeutic strategy.

\section{Introduction}

Gliomas account for $\sim 50 \%$ of all brain tumors $(1,2)$. Among these, malignant gliomas are extremely invasive and are rarely removable by en bloc resection. Despite the use of aggressive therapies, including radical resection, irradiation and chemotherapy, the median survival time of the most malignant type of glioma, glioblastoma multiforme (GBM), is $~ 1$ year from the initial diagnosis $(3,4)$. Temozolomide $(8$-carbamoyl-3-meth ylidazo(5,1-d)-1,2,3,5-terrazin-4(3H)-one; TMZ) has emerged as a well-tolerated, orally administered alkylating agent, delivering a methyl group to DNA purine bases $\left(\mathrm{O}^{6}\right.$-guanine; $\mathrm{N}^{7}$-guanine and $\mathrm{N}^{3}$-adenine), and has the ability to cross the blood-brain barrier and thereby treat primary and recurrent gliomas $(5,6)$. However, its relatively short half-life $(\sim 1.8 \mathrm{~h})$ and the presence of methylguanine-DNA methyltransferase within the tumor result in a high recurrence rate following TMZ-based monotherapy (7). Novel strategies devised to enhance response and thwart resistance are, therefore, the focus of clinical investigation, and are essential for the future improvement of patient prognosis $(8,9)$.

Previous studies have reported that glioma cells respond to TMZ treatment with autophagy, referred as type II programmed cell death (10-12). Notably, Kanzawa et al (13) demonstrated that 3-methyladenine (3-MA) inhibits autophagy through the inhibition of microtubule-associated protein 1 light chain 3 (LC3) localization to the autophagosomal membrane, thereby attenuating glioblastoma cell death. However, the role of autophagy is dependent on cellular context; apart from a cytotoxic role during TMZ action, autophagy induction following mild or moderate cellular stress (for example, starvation) is a cytoprotective process that eliminates stress-reactive cytoplasmic aggregates, macromolecules and organelles in mammalian cells by the lysosomal system and, in turn, supplies energy to the cells to maintain homeostasis through these catabolic phenomena (14-16). Such paradoxical roles of autophagy indicate that an autophagy activator may 
exert antitumor effects when applied in combination with TMZ, causing increased glioblastoma cell autophagy, whilst exerting no detrimental effects or even being beneficial to normal tissue.

The hormonally active form of vitamin D (VD), 1,25-dihydroxycholecalciferol, has well-established actions on autophagy and has low toxicity at low concentrations $(<1,000 \mu \mathrm{g} / \mathrm{day}$, causing conditions such as hypercalcemia only when administered in excess) (17). The present study thus aimed to investigate the potential of using VD as a chemosensitizing agent in glioblastoma treatment. The induction of autophagy by VD relies on an increase in cytoplasmic $\mathrm{Ca}^{2+}$ concentration, which may result from VD receptor-mediated changes to calcium-regulating protein expression. An increase in cytoplasmic $\mathrm{Ca}^{2+}$ concentration activates $\mathrm{Ca}^{2+} /$ calmodulin-dependent kinase kinase- $\beta$, which is followed by the activation of AMP-activated kinase (AMPK), a well-known potent inducer of autophagy (18). AMPK activation induces autophagy via the inhibition of mammalian target of rapamycin complex 1, the major gatekeeper of mammalian autophagy, and the subsequent activation of several autophagy-associated proteins $(19,20)$.

The present study aimed to determine the anticancer effect of VD and TMZ in the co-treatment of glioblastoma, and to identify the underlying mechanism of action. Using the C6 glioblastoma cell line, the in vitro anticancer effects of TMZ and VD were compared with those of TMZ alone through a cell viability assay. In accordance with a previous study, which demonstrated that $100 \mathrm{nM}$ VD could trigger autophagy in breast tumor cells without any signs of apoptotic morphology (21), a $100 \mathrm{nM}$ concentration of VD was used in the present study. Western blotting, flow cytometry, transmission electron microscopy (TEM) and immunofluorescence (IF) were also performed to identify whether autophagy enhancement was the underlying mechanism of this anticancer effect. Finally, these treatments were applied to rat orthotopic xenograft models to determine their antitumor effects in vivo.

\section{Materials and methods}

Cell culture. The C6 rat glioblastoma cell line was purchased from the American Type Culture Collection (ATCC, Manassas, VA, USA). Cells were cultured under sterile conditions, at $37^{\circ} \mathrm{C}$, in a humid environment containing $5 \% \mathrm{CO}_{2}$; culture medium consisted of Dulbecco's modified Eagle's medium supplemented with $10 \%$ fetal bovine serum, $4 \mathrm{mM}$ glutamine, $100 \mathrm{U} / \mathrm{ml}$ penicillin and $100 \mathrm{mg} / \mathrm{ml}$ streptomycin (all purchased from Gibco; Thermo Fisher Scientific, Inc., Waltham, MA, USA). The cultures were regularly checked and trypsinized when cells reached $85 \%$ confluence.

3-(4,5-Dimethylthiazol-2-yl)-2,5-diphenyltetrazolium bromide (MTT) assay. In order to determine the half-maximal inhibitory concentration $\left(\mathrm{IC}_{50}\right.$ value) of $\mathrm{TMZ}, 5 \times 10^{3} \mathrm{C} 6$ cells in single-cell suspensions were seeded into individual wells of 96-well plates and incubated for $24 \mathrm{~h}$ at $37^{\circ} \mathrm{C}$ prior to treatment with TMZ $(0.1,0.5,1,5$ or $10 \mu \mathrm{M}$; Sigma-Aldrich, St. Louis, MO, USA) diluted in dimethyl sulfoxide (DMSO; Gibco; Thermo Fisher Scientific, Inc.) for $24 \mathrm{~h}$ (data not shown). Following determination of the $\mathrm{IC}_{50}$ value of $\mathrm{TMZ}$ as $1 \mathrm{mM}$, cells were treated for $24 \mathrm{~h}$ with DMSO alone, $1 \mathrm{mM} \mathrm{TMZ}$, $100 \mathrm{nM}$ VD (Tocris Bioscience, Ellisville, MO, USA) or a combination of the two ( $1 \mathrm{mM}$ TMZ plus $100 \mathrm{nM}$ VD). To reveal the role of autophagy on C6 cell cytotoxicity, which may be induced by various treatments as mentioned above, cells were treated with $1 \mathrm{mM} \mathrm{3-methyladenine} \mathrm{(3-MA;}$ Sigma-Aldrich), an autophagy inhibitor, at 30 min treatments. MTT solution (Thermo Fisher Scientific, Inc.) was added to each well and the plate was incubated for $4 \mathrm{~h}$ at $37^{\circ} \mathrm{C}$ prior to removing the culture medium. DMSO was then added for $30 \mathrm{~min}$ at room temperature. Cell viability was determined using a spectrophotometer by measuring the absorbance at 492 nm (Ultrospec 7000; Biochrom, Holliston, MA, USA). Viability for each group was calculated as a percentage of that of the control group.

Clonogenic assay. Prior to plating, the cell culture medium was removed and the cells were washed twice with phosphate-buffered saline (PBS). Adherent cells were then trypsinized and counted. A total of 5,000 cells were seeded into $60-\mathrm{mm}$ tissue culture dishes (Greiner Bio-One GmbH, Frickenhausen, Germany) containing culture medium supplemented with DMSO, $1 \mathrm{mM}$ TMZ, $100 \mathrm{nM}$ VD or a combination of $1 \mathrm{mM}$ TMZ and $100 \mathrm{nM}$ VD. Colonies were allowed to form for $72 \mathrm{~h}$. The cell culture medium was then removed and the cells were washed twice with PBS. Colonies were fixed using 100\% methanol for $30 \mathrm{~min}$ at room temperature, and stained with Coomassie Blue (Thermo Fisher Scientific, Inc.) for $15 \mathrm{~min}$. The number of colonies containing $\geq 50$ cells was counted. This experiment, and the following assays were performed in triplicate.

Wound healing assay. Wound healing experiments were performed, as previously described (22). Briefly, the cells were seeded on $60-\mathrm{mm}$ tissue culture dishes and grown to confluence. The cells were treated with DMSO, TMZ, VD or a combination of TMZ and VD, and scratched with a sterile $20-\mu \mathrm{l}$ pipette tip to create an artificial wound. Immediately and $24 \mathrm{~h}$ after wounding, the wound healing process was imaged using an inverted microscope with a $10 \mathrm{X}$ long-working-distance objective (1X51; Olympus Corporation, Tokyo, Japan). Cell migration was quantified by measuring the diameter of the wound at 0 and $24 \mathrm{~h}$ using the image analyzing software, ImageJ (version 1.45; NIH, Bethesda, MA, USA).

Hoechst 33258 staining. In order to evaluate levels of apoptosis, $1 \times 10^{6}$ cells/well were plated on coverslips (BD Biosciences, Bedford, MA, USA) in a 12-well plate. The cells were treated with DMSO, TMZ, VD or a combination of TMZ and VD and kept in a $\mathrm{CO}_{2}$ incubator at $37^{\circ} \mathrm{C}$ for $24 \mathrm{~h}$. Following incubation and addition of fresh medium, the cells were incubated with $5 \mu 1$ Hoechst 33258 (Sigma-Aldrich) per well at $37^{\circ} \mathrm{C}$ for $10 \mathrm{~min}$. The number of apoptotic cells was then assessed using an LSM 700 laser confocal microscope (Zeiss, Oberkochen, Germany). Increased fluorescence with shrunken nuclei was indicative of apoptotic cells, whereas weak fluorescence with normally-sized nuclei was indicative of non-apoptotic cells. The number of apoptotic cells was quantified by capturing images in random fields and counting $\geq 200$ cells from 4 random fields in each well. 
IF staining. LC3 is a reliable marker for cells undergoing autophagy (23). A total of $1 \times 10^{6}$ cells/well were seeded onto sterile glass coverslips. After a $24 \mathrm{~h}$ treatment with DMSO, TMZ, VD or a combination of TMZ and VD, cells were fixed using 4\% paraformaldehyde (Sigma-Aldrich), blocked with $3 \%$ normal goat serum (Dako, Carpinteria, CA, USA) and incubated in $1 \%$ bovine serum albumin (Sigma-Aldrich) $/ 10 \%$ normal goat serum/0.3 M glycine in $0.1 \%$ PBS-Tween for $1 \mathrm{~h}$ to permeabilize the plasma membrane and to block non-specific protein-protein interactions. The cells were then incubated with a rabbit polyclonal anti-LC3 antibody (1:500; ab128025; Abcam, Cambridge, UK) for 2 h. Cells were washed twice with PBS, incubated for an additional $30 \mathrm{~min}$ with fluorescein isothiocyanate-conjugated anti-rabbit IgG antibody $(1: 1,000$; ab6717; Abcam), washed again with PBS and mounted onto slides with 4',6-diamidino-2-phenylindole-conjugated mounting medium (Abcam). Following image acquisition with the LSM-700 laser confocal microscope, LC3 puncta in cells were quantified using 10 randomly-selected images from each drug treatment group.

Flow cytometry. Cells were analyzed for the presence of LC3 using a FACSCanto II flow cytometer (BD Biosciences) according to the manufacturer's protocol. Following 2 washes with PBS, cells were fixed using $4 \%$ paraformaldehyde for $10 \mathrm{~min}$ at $37^{\circ} \mathrm{C}$ and permeabilized with $0.25 \%$ Triton $\mathrm{X}-100$ in PBS for $10 \mathrm{~min}$. Cells were stained with rabbit polyclonal anti-LC3 antibody (1:100; ab128025; Abcam, Cambridge, $\mathrm{UK})$ for $1-2 \mathrm{~h}$ at $4^{\circ} \mathrm{C}(1: 100)$ and goat anti-rabbit $\mathrm{IgG}$ antibody (1:200; ab6717; Abcam) for $1 \mathrm{~h}$ on ice. Following 2 additional washes with PBS, cells were fixed using $4 \%$ paraformaldehyde and assayed immediately. Flow cytometry data were collected using 10,000-30,000 cells and were analyzed using FlowJo software (version 10.1; Tree Star, Ashland, OR, USA).

TEM. Cells were collected and fixed in $2 \%$ paraformaldehyde and $0.1 \%$ glutaraldehyde in $0.1 \mathrm{M}$ sodium cacodylate for $2 \mathrm{~h}$, post-fixed with $1 \% \mathrm{OsO}_{4}$ for $1 \mathrm{~h}, \mathrm{PBS}$-washed and stained for $1 \mathrm{~h}$ in $3 \%$ aqueous uranyl acetate. The cells were washed again, dehydrated with graded alcohol and embedded in Epon-Araldite resin (Canemco Inc., Gore, QC, Canada). Ultrathin sections were cut using an ultramicrotome (Reichert, Inc., Depew, NY, USA), counterstained with $0.3 \%$ lead citrate and examined using an HT7700 transmission electron microscope (Hitachi, Ltd., Tokyo, Japan).

Immunoblotting. C6 cells treated with vehicle, TMZ, VD or a combination of TMZ and VD were lysed using a lysis buffer containing $20 \mathrm{mM}$ Tris- $\mathrm{HCl}$ (pH 8.0), $150 \mathrm{mM} \mathrm{NaCl}, 2 \mathrm{mM}$ EDTA, $100 \mathrm{mM} \mathrm{NaF}, 1 \mu \mathrm{g} / \mathrm{ml}$ leupeptin, $1 \mu \mathrm{g} / \mathrm{ml}$ antipain and $1 \mathrm{mM}$ phenylmethylsulfonyl fluoride. The protein content in the cell lysates was determined using a BCA protein assay kit (Thermo Fisher Scientific, Inc.). Immunoblotting was conducted by resolving $30-50 \mu \mathrm{g}$ protein by $15 \%$ sodium dodecyl sulfate-polyacrylamide gel electrophoresis and electroblotting onto polyvinylidene difluoride membranes for western blot analysis. Blots were probed with the following 1:1,000-diluted primary rabbit antibodies: Anti-cleaved caspase-3 (ab32042), $\beta$-actin (ab189073), LC3 (ab128025), beclin-1 (ab55878) and p62 (ab91526) overnight at $4^{\circ} \mathrm{C}$, followed by incubation with horseradish peroxidase-conjugated goat anti-rabbit IgG (1:1,000; ab6717) secondary antibody for $1 \mathrm{~h}$ at room temperature. The proteins were then visualized by an enhanced chemiluminescence system (Thermo Fisher Scientific, Inc.) with exposure to X-ray film. Finally, the blots were scanned and densitometric analysis was performed using Scion Image (Beta 4.02 release) software (Scion Corporation, Torrance, CA, USA).

Experimental animals. Male Sprague-Dawley rats were obtained from the Experimental Animal Center, Konyang University (Daejeon, South Korea). A total of 24 male rats (age, 2-months; body weight, 250-300 g) were used. The animals were housed at $23^{\circ} \mathrm{C}$ and $60 \%$ relative humidity, with a 12:12 h light:dark cycle and free access to water and food. Animal handling and care conformed to the Konyang University institutional guidelines, which comply with international law and policy (as described in the NIH Guide for the Care and Use of Laboratory Animals; NIH Publication No. 85-23, 1985, revised 1996). The study was approved by the ethics committee of Konyang University, Daejeon, Korea). All experiments were designed to minimize the number of animals used and the detriment to the animals' mental and physical wellbeing.

Rat orthotopic xenograft model. To establish the rat glioblastoma model, the cell implantation procedure was conducted based on the method developed by Kobayashi et al (24). Briefly, each animal was anesthetized (ketamine 40-90 mg/kg, intraperitoneally and xylazine $5-10 \mathrm{mg} / \mathrm{kg}$, subcutaneously; both purchased from Daihan Pharmaceutical Co., Ltd., Seoul, Korea) and immobilized on a stereotaxic unit (Stoelting Co., Wood Dale, IL, USA). Following disinfection and incision of the skin with a scalpel, a hole was drilled through the skull 2-mm lateral and 2-mm anterior to the bregma, on the right side of the skull. A total of $1 \times 10^{6} \mathrm{C} 6$ cells, resuspended in $10 \mu \mathrm{l}$ saline solution, were injected with a 25 -gauge Hamilton syringe (Hamilton, Reno, NV, USA) at a 3-mm depth from the dura, at a rate of $2 \mu \mathrm{l} / \mathrm{min}$. A waiting time of $2 \mathrm{~min}$ was implemented following injection to avoid leakage. At post-operative day 7 (POD 7), animals were divided into three groups ( $n=8$ animals per group). The first group was treated with $200 \mu 1 \mathrm{DMSO}$, the second group was treated intraorally (i.o.) with $10 \mathrm{mg} / \mathrm{kg} /$ day $\mathrm{TMZ}$, dissolved in $200 \mu \mathrm{l}$ DMSO, and the third group received a subcutaneous (s.c.) injection of $0.2 \mu \mathrm{g} / \mathrm{kg} /$ day of VD dissolved in $200 \mu \mathrm{l}$ of saline solution and also received TMZ as described above. The treatment duration was 1 week. Magnetic resonance imaging (MRI) was used to evaluate tumor size in vivo. Prior to imaging, rats were anesthetized, using the aforementioned anesthetics and doses, and placed in the imaging chamber of a 7T/30 cm MRI scanner (Pharmascan 7T; Bruker BioSpin $\mathrm{GmbH}$, Karlsruhe, Germany). The parameters of the scan were as follows: Field of view, $60 \mathrm{~mm}$; slice thickness, $0.5 \mathrm{~mm}$; multiple echo times, $35.1 \mathrm{msec}$; and repetition time, $3,500 \mathrm{msec}$. Prior to drug treatment, the confirmation of successful modeling was conducted by T2-weighted MRI at POD 7. At the end of the 7-day treatment period, on POD 14, tumors from each rat were imaged again by MRI to compare the antitumor effects of the drug treatments. A total of $20 \mathrm{MRI}$ images per animal were obtained and tumor volume 
A

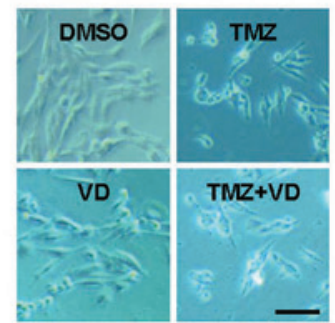

C

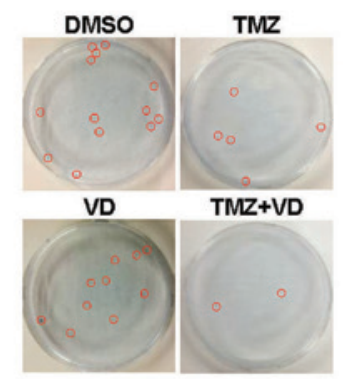

B

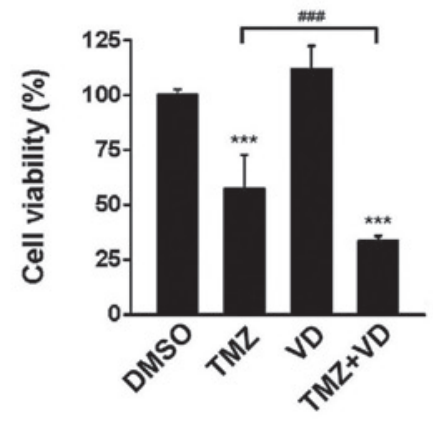

D

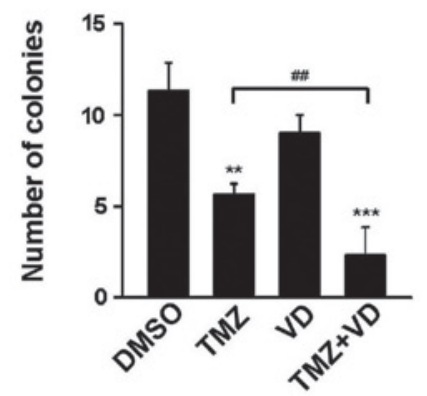

$\mathbf{E}$

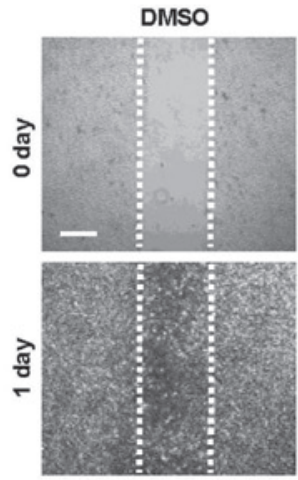

TMZ

VD

$\mathrm{TMZ}+\mathrm{VD}$
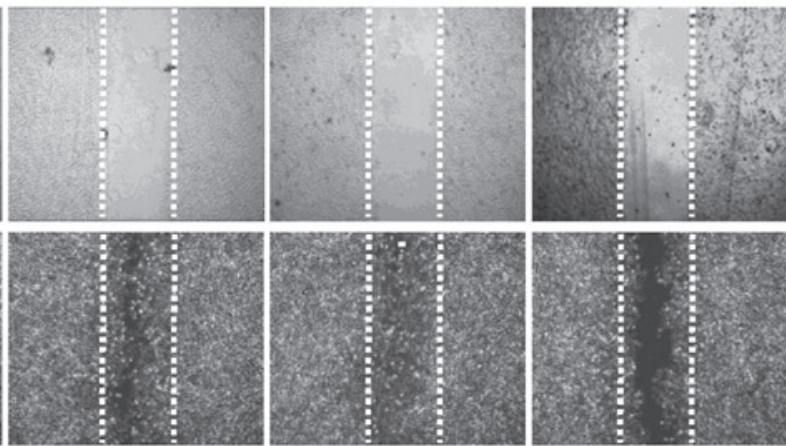

$\mathbf{F}$

$\square 0$ day treatment $\square 1$ day treatment

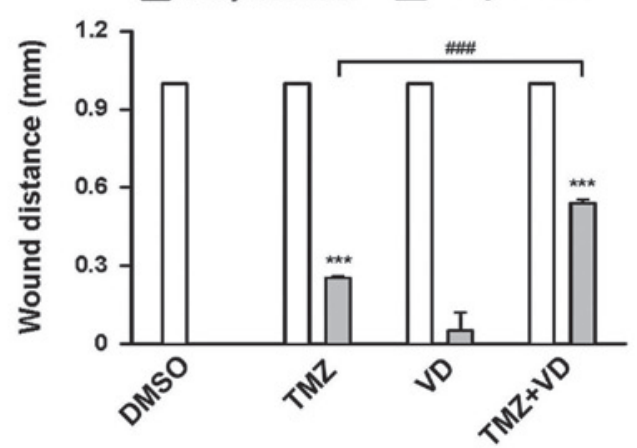

Figure 1. In vitro effects of TMZ + VD combination therapy in the C6 rat glioblastoma cell line. (A) Representative microscopic images of C6 cells incubated with DMSO, TMZ, VD or TMZ + VD for $24 \mathrm{~h}$. Scale bar represents $100 \mu \mathrm{m}$. (B) Graph of the treatment cytotoxicity, measured by MTT assay. (C) Representative images of colonies formed by C6 cells incubated with DMSO, TMZ, VD or TMZ + VD for 72 h., marked with red circles. (D) Graph of the colony counts for each group. The number of colonies containing $\geq 50$ cells was selectively counted. (E) Representative microscopic images of a wound healing assay using C6 cells treated with DMSO, TMZ, VD or TMZ + VD for $24 \mathrm{~h}$. Scale bar represents $500 \mu \mathrm{m}$. (F) Graph of the wound diameter remaining following treatment, expressed as the mean \pm standard error of the mean of $\geq 3$ independent experiments. ${ }^{* *} \mathrm{P}<0.01$ and ${ }^{* * *} \mathrm{P}<0.001$ vs. DMSO-treated cells; ${ }^{\# \#} \mathrm{P}<0.01$ and ${ }^{\# \# /} \mathrm{P}<0.001$ vs. TMZ-treated cells. DMSO, dimethyl sulfoxide; TMZ, temozolomide; VD, vitamin D; MTT, 3-(4,5-dimethylthiazol-2-yl)-2,5-diphenyltetrazolium bromide.

was measured using 3D-Doctor Software (Able Software Corp, Lexington, MA, USA) with a thresholding method (25).

Statistical analysis. The survival curves of the tumor-bearing rats were estimated according to the Kaplan-Meier method, and the curves were compared using a generalized Wilcoxon test.
One-way analysis of variance tests were performed to detect differences in effects between the treatments. All data are presented as mean \pm standard error of the mean. Comparisons of the data between the groups were performed with Student's t-tests using SPSS software (version IBM Corp., Armonk, NY, USA). Differences with P-values $<0.05$ were considered to be 
A
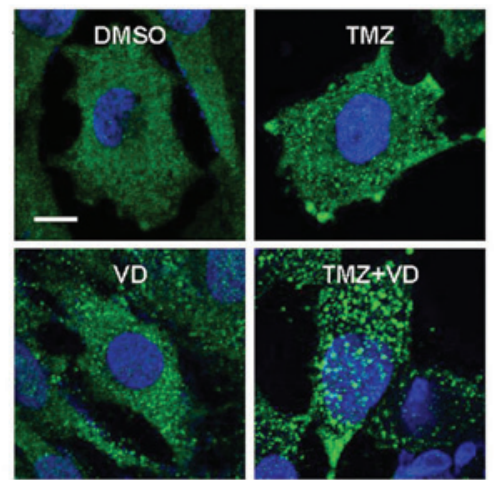

C

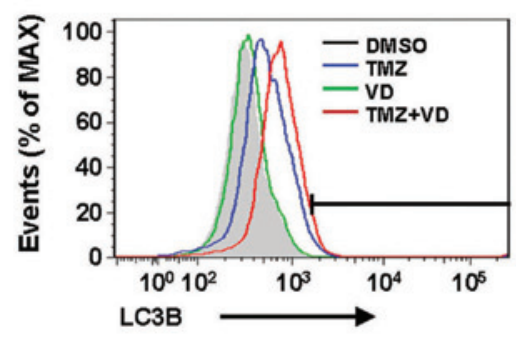

B

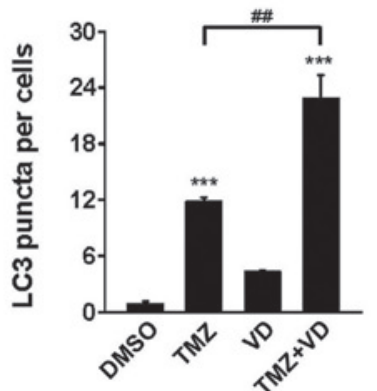

D

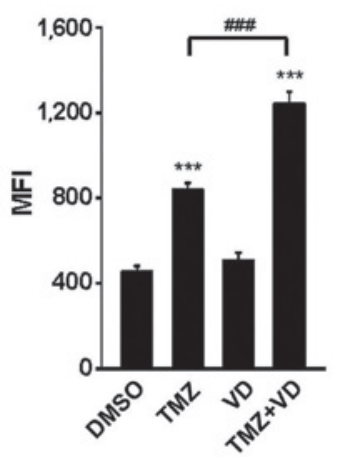

$\mathbf{E}$
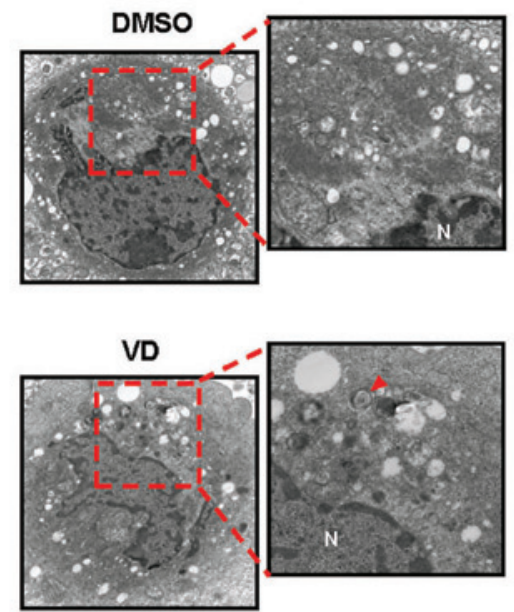

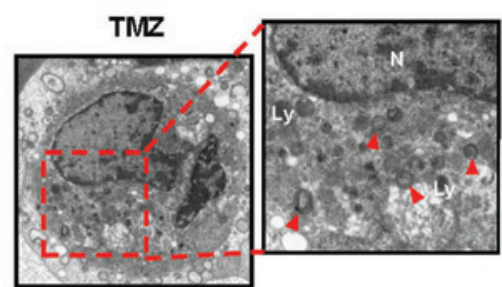

$\mathbf{F}$

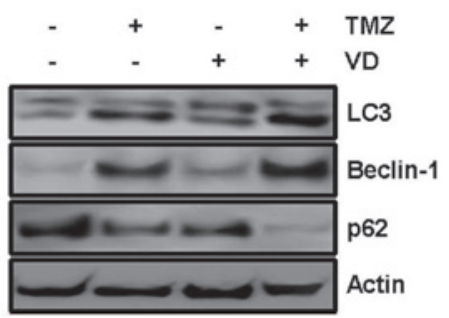

Figure 2. Enhancement of autophagic activity in C6 cells treated with TMZ and VD for $24 \mathrm{~h}$. (A) Representative immunofluorescence images demonstrating LC3 immunoreactivity in C6 cells treated with DMSO, TMZ, VD or TMZ + VD. Scale bar represents $10 \mu \mathrm{m}$. (B) Graph of LC3 puncta quantification, demonstrating the average number of LC3 puncta per cell from 10 randomly selected images. (C) Representative fluorescence-activated cell sorting profiles for LC3 expression in C6 cells treated with DMSO, TMZ, VD or TMZ + VD. (D) Graph of the MFI, expressed as the mean \pm standard error of the mean from $\geq 3$ independent experiments. ${ }^{* * *} \mathrm{P}<0.001$ vs. DMSO-treated cells; ${ }^{\# \#} \mathrm{P}<0.01$ and ${ }^{\# \# \#} \mathrm{P}<0.001$ vs. TMZ-treated cells. (E) Autophagy in transmission electron micrographs (x9,700 magnification) of C6 cells treated with DMSO, TMZ, VD or TMZ + VD. Red arrowheads indicate autophagosomes. (F) Representative immunoblots demonstrating LC3, beclin-1 and p62 expression in C6 cells treated with the indicated drugs. Actin was used as a loading control. DMSO, dimethyl sulfoxide; TMZ, temozolomide; VD, vitamin D; LC3, microtubule-associated protein 1 light chain 3; MFI, mean fluorescence intensity.

statistically significant. Each n-value refers to the number of separate experiments conducted.

\section{Results}

Combination with VD enhances the cytotoxicity of TMZ to a rat glioblastoma cell line in vitro. To examine whether co-treatment with TMZ and VD inhibited the growth of C6 cells, a rat glioblastoma cell line, in vitro experiments were performed. Cells were incubated in a culture medium containing DMSO, VD alone, or TMZ with or without VD, for $24 \mathrm{~h}$. An MTT assay was then performed to compare the cytotoxicity of each treatment. As demonstrated in Fig. 1A and B, treatment with VD alone did not suppress cell growth, but TMZ alone or in combination with TMZ significantly inhibited cell growth $(\mathrm{P}<0.001)$, compared with the DMSO control. Notably, growth was inhibited to a greater extent in cells treated with TMZ and VD when compared with the growth of cells treated with TMZ alone (cell viability, $29.9 \pm 3.7$ vs. $54.2 \pm 14.3 \%$, respectively; $\mathrm{P}<0.001$ ). These data were supported by two additional experiments; TMZ treatment reduced the number of colonies (Fig. 1C and D) and reduced the wound healing (i.e., migratory) ability of the C6 cells (Fig. 1E and F). TMZ and VD co-treatment was 
A
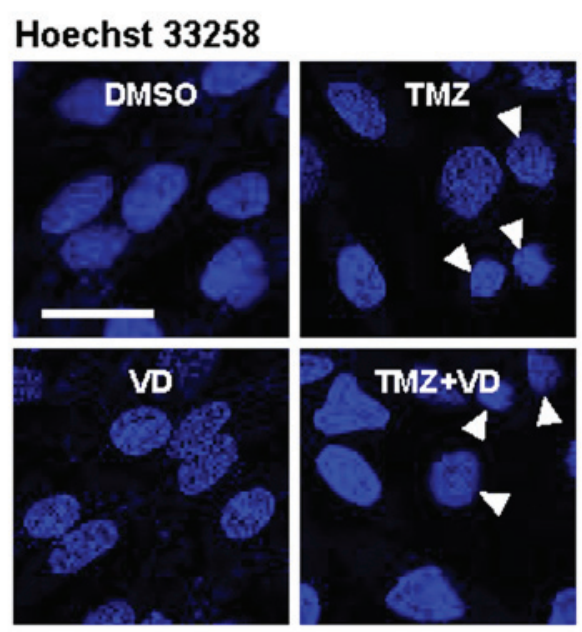

C

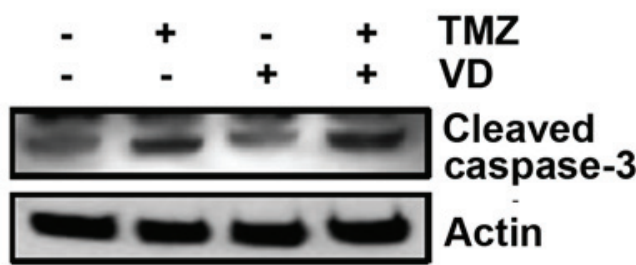

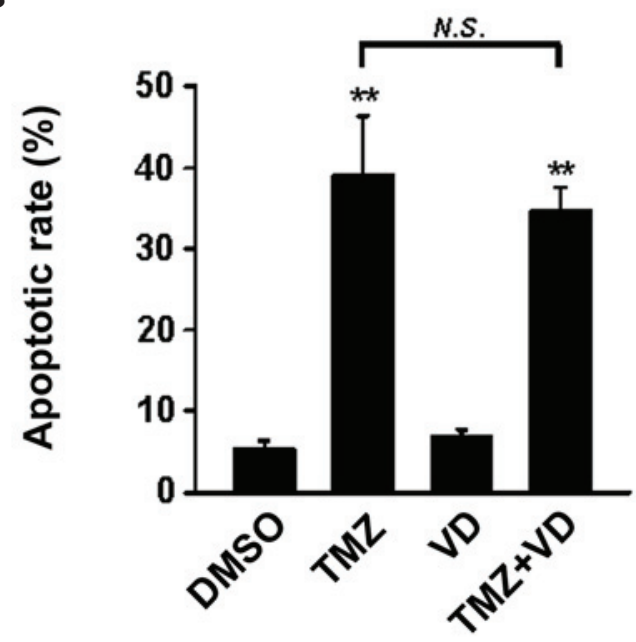

D

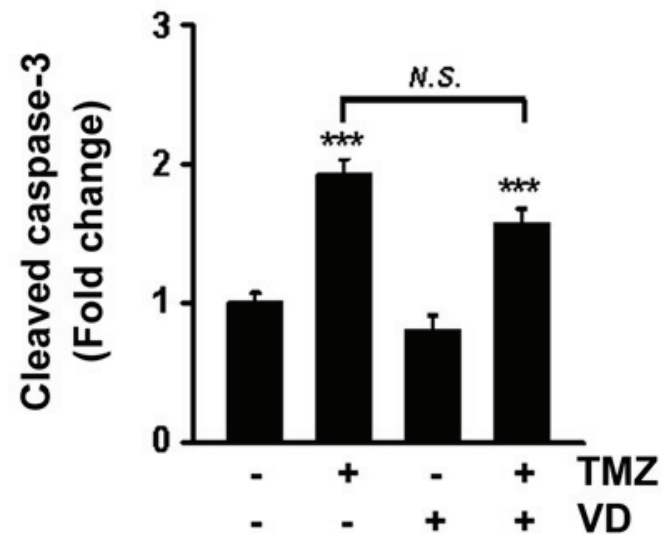

Figure 3. Apoptosis in C6 cells treated with TMZ and VD for 24 h. (A) Representative images demonstrating Hoechst 33258 staining. Shrunken nuclei with intense fluorescence (white arrowheads) were considered to be apoptotic nuclei, and normal-sized nuclei with weak fluorescence were considered to be non-apoptotic. Scale bar represents $150 \mu \mathrm{m}$. (B) Graph of the proportion of apoptotic nuclei to all nuclei in 4 randomly-selected microscopic fields (of $\geq 100$ cells in each field), expressed as a percentage. (C) Representative immunoblots of cleaved caspase-3 expression in C6 cells treated with the indicated drugs. (D) Graph of the densitometric analysis, expressed as the mean fold change vs. the control \pm standard error of the mean from $\geq 3$ independent experiments. Actin was used as a loading control. ${ }^{* *} \mathrm{P}<0.01$ and ${ }^{* * *} \mathrm{P}<0.001$ vs. DMSO-treated cells. DMSO, dimethyl sulfoxide; TMZ, temozolomide; VD, vitamin $\mathrm{D}$; N.S., not significant.

more effective than TMZ alone in reducing colony formation (2.6 \pm 2.2 vs. $5.9 \pm 0.8$ colonies, respectively; $\mathrm{P}<0.01)$ and inhibiting wound healing (wound distance, $0.56 \pm 0.05$ vs. $0.28 \pm 0.03 \mathrm{~mm} ; \mathrm{P}<0.001)$. These data suggest that the combined use of TMZ and VD may be an effective therapy for glioblastoma treatment.

TMZ and VD co-treatment enhances autophagy in glioblastoma cells. TMZ and VD have separately been reported to induce autophagy in numerous cell types $(26,27)$, so the present study examined the induction of autophagy by TMZ, VD or a combination of TMZ and VD in C6 cells. After $24 \mathrm{~h}$ of treatment, cells were collected and stained using IF (Fig. 2A and B) and examined by flow cytometric analysis (Fig. 2C and D) to detect the presence of LC3. LC3 is an established autophagy marker due to its involvement in autophagosome membrane formation $(28,29)$. TEM observation (Fig. 2E) was performed to detect activation of autophagy. All three drug treatments could activate autophagy in C6 cells. In the cells treated with a combination of TMZ and VD compared with the cells treated with $\mathrm{TMZ}$ alone, the estimated mean number of LC3 puncta per cell
(Fig. 2A and B) and mean fluorescent intensity (Fig. 2C and D) were increased almost 2-fold $(22.1 \pm 3.6$ vs. $11.9 \pm 0.2$ puncta; $\mathrm{P}<0.01)$ and 1.5 -fold $(1,210.3 \pm 62.5$ vs. $820.2 \pm 34.4 ; \mathrm{P}<0.001)$, respectively. Ultrastructural observation using TEM (Fig. 2E) revealed the generation of small autophagosomes, double-layered structures engulfing intracellular organelles, in cells treated with TMZ or VD. However, the autophagosome number and size increased in cells treated with TMZ and VD compared with the cells treated with TMZ alone, also confirmed by IF and TEM. The quantitative changes of autophagy-related protein expression in C6 cells were also measured. As demonstrated in Fig. 2F, TMZ treatment induced the conversion of LC3-I to LC3-II (lipidated form), a reliable marker of autophagosome generation. Furthermore, the expression levels of beclin-1 and p62, positive and negative upstream regulators of LC3 recruitment, respectively, were indicative of the induction of autophagy in TMZ-treated cells (30). The alterations of the level of autophagy-related protein expression were significantly increased by the TMZ and VD treatment.

The effects of TMZ and VD co-treatment on apoptosis, morphologically and quantitatively, were also examined (Fig. 3A 


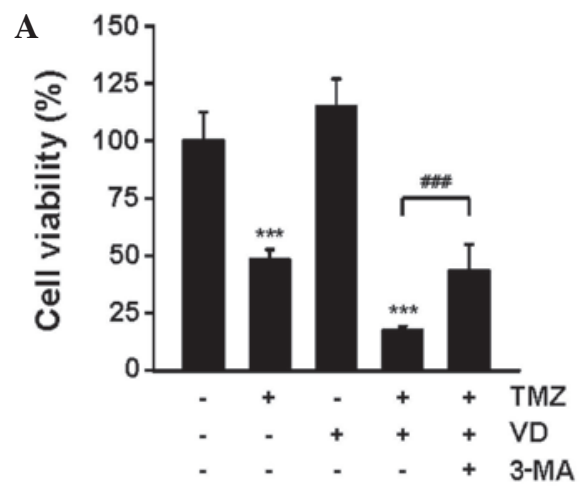

B

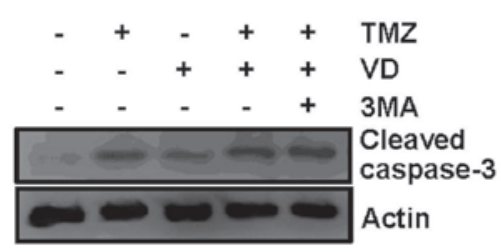

C

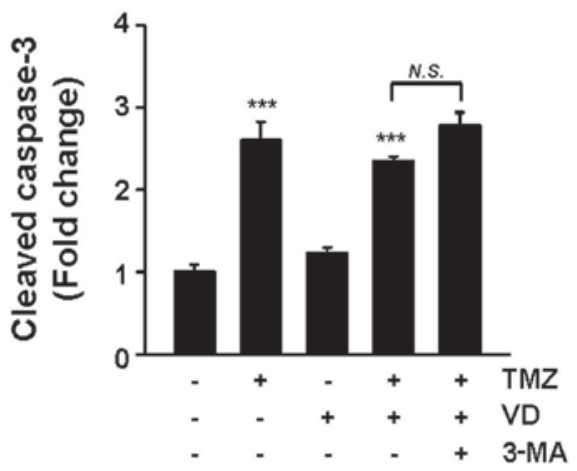

Figure 4. Role of autophagy in the synergistic effect of TMZ and VD on C6 cell death. (A) Graph of the cytotoxic effect of a 24-h TMZ, VD or TMZ + VD treatment of $\mathrm{C} 6$ cells, with or without co-incubation with 3-MA, expressed as percentage of untreated control cells. (B) Representative immunoblots demonstrating cleaved caspase-3 expression in C6 cells treated with the indicated drug combinations. (C) Densitometric analysis of cleaved caspase-3 expression in drug-treated C6 cells, with or without co-incubation with 3-MA, expressed as the mean fold change vs. untreated control cells \pm standard error of the mean from $\geq 3$ independent experiments. Actin was used as a loading control. ${ }^{* * *} \mathrm{P}<0.001$ vs. untreated control cells; ${ }^{\# \#} \mathrm{P}<0.001 \mathrm{vs}$. TMZ + VD-treated cells. TMZ, temozolomide; VD, vitamin D; 3-MA, 3-methyladenine.

A
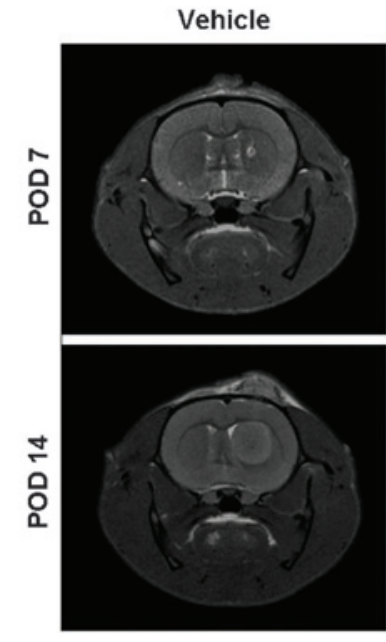

B

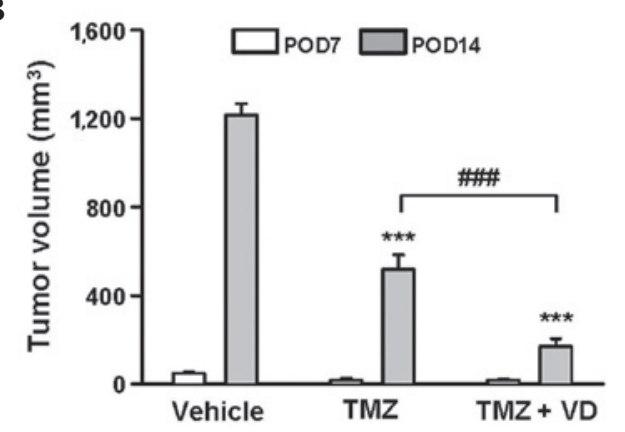

TMZ
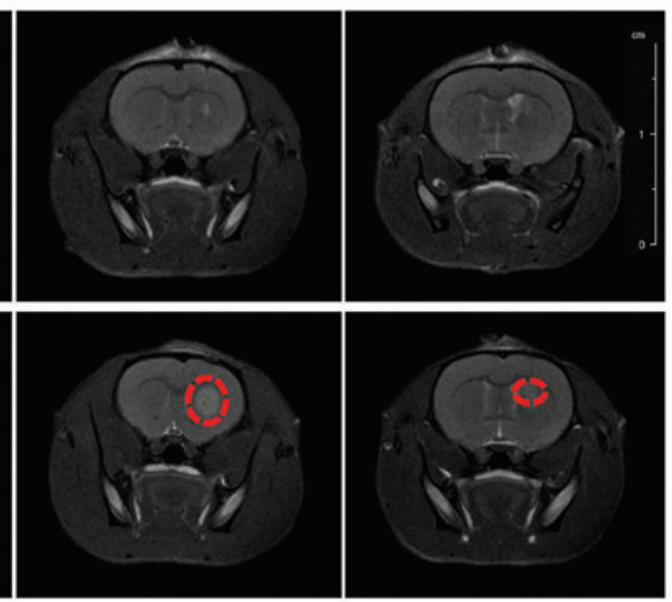

C

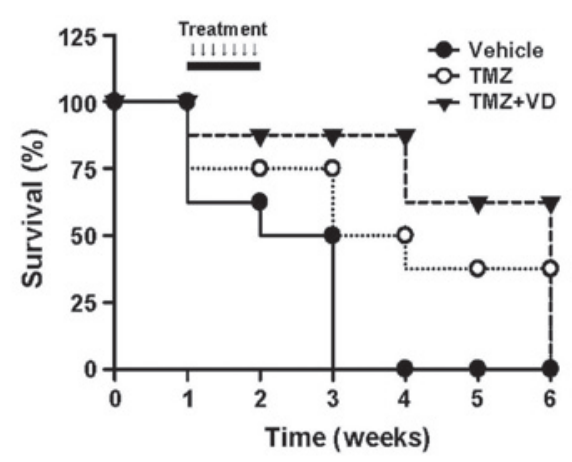

Figure 5. In vivo effects of TMZ and VD combination therapy on tumor size regression and prolonged survival in orthotopic, xenografted tumor-bearing rats $(\mathrm{n}=8)$. (A) Representative T2-weighted magnetic resonance images, used to monitor brain tumor progression at POD 7 and 14 in rats treated for 7 days (from POD 8 to POD 14) with vehicle, TMZ (10 mg/kg/day, i.o.) or TMZ (10 mg/kg/day, i.o.) + VD (0.2 $\mu \mathrm{g} / \mathrm{kg} / \mathrm{day}$, s.c.). Red circles represent comparative tumor size between the TMZ- and TMZ + VD-treated groups. (B) Graph of tumor volumetric analysis, expressed as the mean \pm standard error of the mean. ${ }^{* * * *} \mathrm{P}<0.001$ vs. vehicle-treated rats; ${ }^{\# \# \#} \mathrm{P}<0.001$ vs. TMZ-treated rats. (C) Kaplan-Meier survival curves. Rats with intracranial C6 cells were observed for 6 weeks post-treatment; the arrows indicate the timing of the 7-day scheduled regimen for each treatment. TMZ, temozolomide; VD, vitamin D; POD, post-operative day; i.o., intraorally; s.c., subcutaneously.

and B). Following staining with Hoechst 33258, small, bright, condensed nuclei occurred at a significantly greater frequency in cells treated with TMZ alone or TMZ and VD (39.1 18.3 and $35.0 \pm 3.4 \%$, respectively), compared with DMSO-treated cells
$(\mathrm{P}<0.01)$. Furthermore, western blot analysis to quantify cleaved (active-) caspase-3, the most important effector of the apoptotic process, supported increased apoptosis following these treatments (Fig. 3C and D). Notably, no significant difference in 
apoptosis was identified between the TMZ-treated and the TMZ plus VD-treated cells. The present results suggest that TMZ and VD co-treatment enhanced the autophagic process, but not apoptosis, in glioblastoma cells.

Involvement of cytotoxic autophagy in the synergistic effect of $V D$ and TMZ. The role of autophagy induced by the TMZ and VD co-treatment on tumor suppression was elucidated in the current study through use of the autophagy inhibitor 3-MA. As demonstrated in Fig. 4A, 3-MA treatment significantly attenuated the tumoricidal activity of the TMZ and VD co-treatment (cell viability, $25.4 \pm 8.1$ vs. $12.2 \pm 2.5 \%$ in TMZ and VD-treated cells; $\mathrm{P}<0.001)$. Addition of $3-\mathrm{MA}$ caused $\mathrm{C} 6$ cell survival rates to be restored to those of C6 cells treated with TMZ alone. The evaluation of caspase-3 expression levels using western blot analysis revealed that 3-MA had no significant effect on apoptosis (Fig. 4B and C). These results indicate that the antitumor effect of the TMZ and VD co-treatment is due, at least in part, to the enhancement of autophagy, without affecting apoptosis.

Synergistic effect of VD in combination of TMZ in the in vivo rat glioblastoma model. The growth dynamics of xenografted C6 tumors were analyzed by MRI. At POD 7 in all tumor-bearing rats, the glioblastoma presented as a hyperintensive tumor in the T2-weighted images; no significant difference was observed in tumor size between the experimental groups. In contrast to vehicle treatment, the administration of TMZ alone and of TMZ plus VD for 1 week significantly inhibited tumor growth (Fig. 5A), which reached $580 \pm 83$ and $186 \pm 52 \mathrm{~mm}^{3}$ in rats treated with $\mathrm{TMZ}$ alone or $\mathrm{TMZ}$ plus VD, respectively. These results suggest a synergism between TMZ and VD (Fig. 5B). Tumor-bearing rats treated with TMZ plus VD demonstrated a significantly prolonged survival duration (Fig. 5C). The median survival duration was 4 weeks in rats treated with TMZ only, but $\geq 5$ weeks for rats co-treated with TMZ and VD. These data together indicate that treatment with a combination of TMZ and VD enhanced TMZ therapeutic efficacy against glioblastoma in vivo.

\section{Discussion}

The current study established that a combination of VD and $\mathrm{TMZ}$ may have therapeutic potential in the treatment of GBM, and provided evidence that chemosensitization to TMZ by VD occurs through the enhancement of autophagy. Considering that GBM cells commonly carry mutations that inactivate the apoptotic pathway $(31,32)$, the enhancement of autophagy by TMZ and VD combination therapy could represent an alternative method for the elimination of GBM cells. However, the mechanism by which autophagy enhancement kills GBM cells, and whether the enhancement of autophagy is the only mechanism involved in the action of TMZ and VD against GBM, remain to be determined.

Autophagy is the principal cellular route used for degrading long-lived proteins and cytoplasmic organelles, and the catabolic advantage of increased autophagy may be critical in stress conditions $(33,34)$. Induced autophagy may, therefore, reflect an adaptive mechanism used to prevent cell death. A marked correlation between reduced autophagy and cancer has previously been reported $(35,36)$. A previous study has also indicated that several proteins and signaling pathways that are associated with autophagy are deregulated during malignant transformation, resulting in reduced autophagic activity; this previous study demonstrated that cytoplasmic levels of beclin-1 protein and mRNA were lower in GBMs compared with lower-grade astrocytomas and normal brain tissue (37). Another previous study involving biochemical analysis of biopsied tumor samples reported that high cytoplasmic levels of the protein beclin-1 positively correlated with the survival and performance status of patients, whereas a low expression level of beclin-1 correlated with an increase in cell proliferation (38). Furthermore, high LC3 expression has been associated with improved survival in GBM patients with poor performance scores (39). Based on these previous reports and the current data, restoration of autophagic activity to normal levels in GBM cells, which is commonly downregulated, may be a potential strategy to treat GBM, and could serve as a mechanism for the restriction of uncontrolled tumor cell growth.

In addition to the hypothesis of 'autophagy restoration' as a mechanism underlying the autophagy-induced antitumor effect, there may be another explanation; during normal autophagy, specific cytoplasmic constituents are isolated from the rest of the cell within an autophagosome, which then fuses with a lysosome and its cargo is degraded and recycled (40-42). When autophagy is upregulated, the rates of autophagosome formation exceed the rates of lysosomal degradation, a condition termed 'autophagic stress', is generated in the cell. If stress or dysregulated autophagy persist, cell death may occur through energy depletion or through alteration of the beclin-1/bcl-2 balance. The cells may also apoptose due to the hyperactivity of autophagosomes, engulfing vital cytoplasmic organelles, including the mitochondria and the endoplasmic reticulum. It has been suggested that the overall autophagic activity is increased in a cell predisposed to death, when compared with the normal cytoplasmic and organelle turnover occurring in healthy cells. As a consequence, the cell 'cannibalizes' itself from inside, a key feature of type II programmed cell death. Although the precise pathway by which VD increases the therapeutic efficacy of TMZ against GBM remains to be elucidated, this combination treatment may exert its antitumor effects by utilizing the two aforementioned autophagy mechanisms.

VD has multiple modes of action, which indicates that there may be other mechanisms underlying its synergism with TMZ besides the autophagy enhancement demonstrated in the present study; previous studies have demonstrated that VD exerts an antitumor effect by interfering with the transduction pathways of growth factor-activated receptors (receptor tyrosine kinases). This subsequently changes transcription and alters genomic functions, resulting in the inhibition of cell proliferation (43). VD has also been proposed to promote angiogenesis (44) and to increase the level of an endogenous protein, cystatin D, which possesses antitumor and anti-metastatic properties (45). Furthermore, VD has been reported to facilitate cancer cell apoptosis by upregulating proapoptotic p53, p21 and Bax proteins, while downregulating anti-apoptotic bcl-2 protein (46). However, the current study did not identify changes in the number of apoptotic nuclei (Fig. 3A and B) or in cleaved caspase-3 expression levels (Figs. 3C and 4B and C) 
in C6 cells treated with VD alone. This discrepancy regarding the role of VD in apoptosis may be due to a different VD dose regimen, C6 cell sensitivity to VD or differing duration of the experiment.

In summary, to the best of our knowledge, the current study is the first to demonstrate that combining VD with TMZ exerts a synergistic effect on the antitumor activity of TMZ in GBM in vivo and in vitro. Although other mechanisms underlying these synergistic effects remain to be determined, an increase in autophagy was identified as a crucial tumoricidal mechanisms of VD chemosensitization during TMZ-based GBM therapy.

\section{Acknowledgements}

The present study was equally supported by a grant from the Development of Forest Science and Technology (no. S111414L030100) and Korea Research Foundation (no. NRF-2014R1A1A4A03005726).

\section{References}

1. Mahaley MS, Mettlin C, Natarajan N, Laws ER Jr and Peace BB National survey of patterns of care for brain-tumor patients. J Neurosurg 71: 826-836, 1989.

2. Avgeropoulos NG and Batchelor TT: New treatment strategies for malignant gliomas. Oncologist 4: 209-224, 1999.

3. Bower M, Newlands ES, Bleehen NM, Brada M, Begent RJ, Calvert H, Colquhoun I, Lewis P and Brampton MH: Multicentre CRC phase II trial of temozolomide in recurrent or progressive high-grade glioma. Cancer Chemother Pharmacol 40: 484-488, 1997.

4. Yung WK, Prados MD, Yaya-Tur R, Rosenfeld SS, Brada M, Friedman HS, Albright R, Olson J, Chang SM, O'Neill AM, et al: Multicenter phase II trial of temozolomide in patients with anaplastic astrocytoma or anaplastic oligoastrocytoma at first relapse. J Clin Oncol 17: 2762-2771, 1999.

5. Newlands ES, Blackledge GR, Slack JA, Rustin GJ, Smith DB, Stuart NS, Quarterman CP, Hoffman R, Stevens MF and Brampton MH: Phase I trial of temozolomide (CCRG 81045: M\&B 39831: NSC 362856). Br J Cancer 65: 287-291, 1992.

6. Dehdashti AR, Hegi ME, Regli L, Pica A and Stupp R: New trends in the medical management of glioblastoma multiforme: The role of temozolomide chemotherapy. Neurosurg Focus 20: E6, 2006.

7. Zhang J, Stevens MF and Bradshaw TD: Temozolomide: Mechanisms of action, repair and resistance. Curr Mol Pharmacol 5: 102-114, 2012.

8. Begemann M, Kashimawo SA, Lunn RM, Delohery T, Choi YJ, Kim S, Heitjan DF, Santella RM, Schiff PB, Bruce JN and Weinstein IB: Growth inhibition induced by Ro 31-8220 and calphostin $\mathrm{C}$ in human glioblastoma cell lines is associated with apoptosis and inhibition of CDC2 kinase. Anticancer Res 18: 3139-3152, 1998

9. Gagliano N, Moscheni C, Torri C, Donetti E, Magnani I, Costa F, Nowicky W and Gioia M: Ukrain modulates glial fibrillary acidic protein, but not connexin 43 expression, and induces apoptosis in human cultured glioblastoma cells. Anticancer Drugs 18: 669-676, 2007.

10. Kanzawa T, Bedwell J, Kondo Y, Kondo S and Germano IM: Inhibition of DNA repair for sensitizing resistant glioma cells to temozolomide. J Neurosurg 99: 1047-1052, 2003.

11. Takeuchi H, Kondo Y, Fujiwara K, Kanzawa T, Aoki H, Mills GB and Kondo S: Synergistic augmentation of rapamycin-induced autophagy in malignant glioma cells by phosphatidylinositol 3-kinase/protein kinase B inhibitors. Cancer Res 65: 3336-3346, 2005.

12. Takeuchi H, Kanzawa T, Kondo Y and Kondo S: Inhibition of platelet-derived growth factor signalling induces autophagy in malignant glioma cells. Br J Cancer 90: 1069-1075, 2004.

13. Kanzawa T, Germano IM, Komata T, Ito H, Kondo Y and Kondo S: Role of autophagy in temozolomide-induced cytotoxicity for malignant glioma cells. Cell Death Differ 11: 448-457, 2004.
14. Gutierrez MG, Master SS, Singh SB, Taylor GA, Colombo MI and Deretic V: Autophagy is a defense mechanism inhibiting BCG and Mycobacterium tuberculosis survival in infected macrophages. Cell 119: 753-766, 2004.

15. Giovannucci E: Vitamin D and cardiovascular disease. Curr Atheroscler Rep 11: 456-461, 2009.

16. Xie Z and Klionsky DJ: Autophagosome formation: Core machinery and adaptations. Nat Cell Biol 9: 1102-1109, 2007.

17. Campbell GR and Spector SA: Hormonally active vitamin D3 (1alpha,25-dihydroxycholecalciferol) triggers autophagy in human macrophages that inhibits HIV-1 infection. J Biol Chem 286: 18890-18902, 2011.

18. Picotto G, Liaudat AC, Bohl L and Tolosa de Talamoni N: Molecular aspects of vitamin D anticancer activity. Cancer Invest 308: 604-614, 2012.

19. Høyer-Hansen M and Jäättelä M: AMP-activated protein kinase: A universal regulator of autophagy? Autophagy 3: 381-383, 2007.

20. Woods A, Dickerson K, Heath R, Hong SP, Momcilovic M, Johnstone SR, Carlson $M$ and Carling D: $\mathrm{Ca}^{2+} /$ calmodulin-dependent protein kinase kinase-beta acts upstream of AMP-activated protein kinase in mammalian cells. Cell Metab 2: 21-33, 2005.

21. Hannigan AM and Gorski SM: Macroautophagy: The key ingredient to a healthy diet? Autophagy 5: 140-151, 2009.

22. Zou J, Wang YX, Mu HJ, Xiang J, Wu W, Zhang B and Xie P: Down-regulation of glutamine synthetase enhances migration of rat astrocytes after in vitro injury. Neurochem Int 58: 404-413, 2011.

23. Tanida I, Ueno T, and Kominami E: LC3 and autophagy. Methods Mol Biol 445: 77-88, 2008.

24. Kobayashi N, Allen N, Clendenon NR and Ko LW: An improved rat brain-tumor model. J Neurosurg 53: 808-815, 1980.

25. Rubin JB, Kung AL, Klein RS, Chan JA, Sun Y, Schmidt K, Kieran MW, Luster AD and Segal RA: A small-molecule antagonist of CXCR4 inhibits intracranial growth of primary brain tumors. Proc Natl Acad Sci USA 100: 13513-13518, 2003.

26. Knizhnik AV, Roos WP, Nikolova T, Quiros S, Tomaszowski KH, Christmann M and Kaina B: Survival and death strategies in glioma cells: Autophagy, senescence and apoptosis triggered by a single type of temozolomide-induced DNA damage. PLoS One 8: e55665, 2013.

27. Wilson EN, Bristol ML, Di X, Maltese WA, Koterba K, Beckman MJ and Gewirtz DA: A switch between cytoprotective and cytotoxic autophagy in the radiosensitization of breast tumor cells by chloroquine and vitamin D. Horm Cancer 2: 272-85, 2011.

28. Kabeya Y, Mizushima N, Ueno T, Yamamoto A, Kirisako T, Noda T, Kominami E, Ohsumi Y and Yoshimori T: LC3, a mammalian homologue of yeast Apg8p, is localized in autophagosome membranes after processing. EMBO J 19: 5720-5728, 2000.

29. Mizushima N, Yamamoto A, Hatano M, Kobayashi Y, Kabeya Y, Suzuki K, Tokuhisa T, Ohsumi Y and Yoshimori T: Dissection of autophagosome formation using Apg5-deficient mouse embryonic stem cells. J Cell Biol 152: 657-668, 2001.

30. Xu HD, Wu D, Gu JH, Ge JB, Wu JC, Han R, Liang ZQ and Qin ZH: The pro-survival role of autophagy depends on Bcl-2 under nutrition stress conditions. PLoS One 8: e63232, 2013.

31. Kapoor GS and O'Rourke DM: Mitogenic signaling cascades in glial tumors. Neurosurgery 52: 1425-1435, 2003.

32. Lefranc F, Brotchi J and Kiss R: Possible future issues in the treatment of glioblastomas: Special emphasis on cell migration and the resistance of migrating glioblastoma cells to apoptosis. J Clin Oncol 23: 2411-2422, 2005.

33. Mizushima N, Ohsumi Y and Yoshimori T: Autophagosome formation in mammalian cells. Cell Struct Funct 27: 421-429, 2002.

34. Levine B, Mizushima N and Virgin HW: Autophagy in immunity and inflammation. Nature 469: 323-335, 2011.

35. Feng Y, Ke C, Tang Q, Dong H, Zheng X, Lin W2, Ke J, Huang J, Yeung SC, and Zhang H: Metformin promotes autophagy and apoptosis in esophageal squamous cell carcinoma by downregulating Stat3 signaling. Cell Death Dis 5: e1088, 2014.

36. Liu YQ, Cheng X, Guo LX, Mao C, Chen YJ, Liu HX, Xiao QC, Jiang S, Yao ZJ, and Zhou GB: Identification of an annonaceous acetogenin mimetic, AA005, as an AMPK activator and autophagy inducer in colon cancer cells. PLoS One 7:e47049, 2012. 
37. Miracco C, Cosci E, Oliveri G, Luzi P, Pacenti L, Monciatti I, Mannucci S, De Nisi MC, Toscano M, Malagnino V, et al: Protein and mRNA expression of autophagy gene Beclin 1 in human brain tumours. Int J Oncol 30: 429-436, 2007.

38. Huang X, Bai HM, Chen L, Li B and Lu YC: Reduced expression of LC3B-II and Beclin 1 in glioblastoma multiforme indicates a down-regulated autophagic capacity that relates to the progression of astrocytic tumors. J Clin Neurosci 17: 1515-1519, 2010.

39. Aoki H, Kondo Y, Aldape K, Yamamoto A, Iwado E, Yokoyama T, Hollingsworth EF, Kobayashi R, Hess K, Shinojima N, et al: Monitoring autophagy in glioblastoma with antibody against isoform B of human microtubule-associated protein 1 light chain 3. Autophagy 4: 467-475, 2008.

40. Jain MV, Paczulla AM, Klonisch T, Dimgba FN, Rao SB, Roberg $\mathrm{K}$, Schweizer F, Lengerke C, Davoodpour P, et al: Interconnections between apoptotic, autophagic and necrotic pathways: Implications for cancer therapy development. J Cell Mol Med 17: 12-29, 2013.

41. Rubinsztein DC, Shpilka T and Elazar Z: Mechanisms of autophagosome biogenesis. Curr Biol 22: 29-34, 2012.
42. Monastyrska I and Klionsky DJ: Autophagy in organelle homeostasis: peroxisome turnover. Mol Aspects Med 27: 483-494, 2006.

43. Audo I, Darjatmoko SR, Schlamp CL, Lokken JM, Lindstrom MJ, Albert DM and Nickells RW: Vitamin D analogues increase p53, p21, and apoptosis in a xenograft model of human retinoblastoma. Invest Ophthalmol Vis Sci 44: 4192-4199, 2003.

44. Bernardi RJ, Johnson CS, Modzelewski RA and Trump DL: Antiproliferative effects of 1alpha,25-dihydroxyvitamin $\mathrm{D}(3)$ and vitamin $\mathrm{D}$ analogs on tumor-derived endothelial cells. Endocrinology 143: 2508-2514, 2002.

45. Alvarez-Díaz S, Valle N, García JM, Peña C, Freije JM, Quesada V, Astudillo A, Bonilla F, López-Otín C and Muñoz A: Cystatin D is a candidate tumor suppressor gene induced by vitamin D in human colon cancer cells. J Clin Invest 119: 2343-2358, 2009.

46. Gorski SM, Chittaranjan S, Pleasance ED, Freeman JD, Anderson CL, Varhol RJ, Coughlin SM, Zuyderduyn SD, Jones SJ, Marra MA: A SAGE approach to discovery of genes involved in autophagic cell death. Curr Biol 13: 358-363, 2003. 